\title{
Numerical modelling of dynamic ductile fracture propagation in different lab-scale experiments using GTN damage model
}

\author{
Benoît Paermentier*, Dimitri Debruyne, Reza Talemi \\ Department of Materials Engineering, KU Leuven, Belgium \\ benoit.paermentier@kuleven.be
}

\begin{abstract}
Initiation and propagation of ductile fractures are major consideration during the design of high-pressure pipelines. Consequences of a pipeline failure can be catastrophic thus structural integrity must be ensured over several decades. Traditional lab-scale experiments such as the Charpy VNotch (CVN) and Drop Weight Tear Test (DWTT), impact experiments on a notched three-point bending sample, are widely used to measure the fracture toughness of a material. However, with increasing wall thickness and the transition to high-grade steels in the pipeline industry, the size-effect of the specimen and inverse fracture became prominent issues. A new testing methodology called the Dynamic Tensile Tear Test (DT3) is currently investigated as to address the issues presented by the current state of the art. In this study, a numerical investigation is conducted on the CVN, DWTT and DT3 experiments to compare the modelling of dynamic ductile fracture propagation in three different testing scales using the Gurson-TvergaardNeedleman (GTN) damage model. X70 and X100 pipeline steel grades are used to model material behaviour. For each considered lab-scale experiment, the dynamic ductile fracture behaviour was successfully reproduced using the GTN damage model.
\end{abstract}

KEYwORDS. Ductile fracture; High strength steel; FEM; Fracture toughness; GTN.

\section{open ACCESS}

Citation: Paermentier, B., Debruyne, D., Talemi, R., Numerical modelling of dynamic ductile fracture propagation in different labscale experiments using GTN damage model, Frattura ed Integrità Strutturale, 52 (2020) 105-112.

Received: 31.10 .2019

Accepted: 17.01 .2020

Published: 01.04.2020

Copyright: (C) 2020 This is an open access article under the terms of the CC-BY 4.0, which permits unrestricted use, distribution, and reproduction in any medium, provided the original author and source are credited.

\section{INTRODUCTION}

$\mathrm{H}$

igh-pressure pipelines are still considered as one of the most efficient means of transport for natural gas and other chemical products. Due to the catastrophic consequences of pipeline failure, structural integrity must be ensured over several decades. Therefore, ductile fracture control is a crucial consideration during the design phase. The high cost of the full-scaled experimental campaigns resulted in the development of more economical lab-scale tests such as the well-known Charpy V-Notch (CVN) impact test, Drop Weight Tear Test (DWTT) [1] and more recently the Dynamic Tensile Tear Test (DT3) [2,3].

Compared to modern pipelines with wall thickness reaching over $20 \mathrm{~mm}$, the CVN has a relatively small standard specimen size with a $10 \mathrm{~mm} \times 10 \mathrm{~mm}$ cross section [4]. Extrapolation of the CVN test data has shown to result in dangerous non- 
conservative predictions since the fracture toughness does not change in proportion to the increasing wall thickness, as has been reported in [5]. In contrast, the DWTT is able to test a full wall thickness specimen which avoided the need for extrapolation. The specimen height increased from $10 \mathrm{~mm}$ to $76.2 \mathrm{~mm}$ and the length increased from $55 \mathrm{~mm}$ to $305 \mathrm{~mm}$. The main differences between the CVN and DW'T test are the larger impacting mass, the larger specimen geometry, and the different striker geometry of the latter. In general, materials are tested using the DWT'T and the Shear Area (SA) criterion is applied to qualify for pipeline applications. However, with the transition to high-grade pipeline steels, issues such as inverse fracture were introduced [6]. Inverse fracture is a phenomenon where a cleavage fracture is initiated in the region impacted by the striker. In the DT3, an even larger specimen with a height of $250 \mathrm{~mm}$ and length of $685 \mathrm{~mm}$ undergoes a tensile load similar to the in-service pipeline conditions.

Numerical modelling approaches are often applied to complement extensive testing campaigns. Based on these correlations, damage models can be validated and/or calibrated in order to be implemented in more complex simulations. The GursonTvergaard-Needleman (GTN) model $[7,8]$ is a well-known and widely applied damage model to simulate dynamic ductile fracture propagation. Due to the limited parameter set, it is often used in industry.

Throughout the development of lab-scale fracture toughness experiments, specimen sizes have been steadily increasing. In this paper the application of the GTN damage model for dynamic fracture propagation in different specimen scales is investigated. The fracture toughness simulations are applied on two high-strength pipeline materials, namely X70 and X100, with different plastic behaviour. The results of the numerical models are used to construct a force-displacement curve to compare experimental data with numerical predictions.

\section{GTN DAMAgE MODEL}

he physical phenomenon of ductile damage can be described using the mechanisms of void nucleation, void growth, and void coalescence $[7,8]$. Due to the limited parameter set, the GTN model has become widely used to model ductile damage behaviour. Based on these mechanisms, the strain based GTN model considers a weakening effect as the plastic deformation increases. The constitutive relations describes the plastic potential $\Phi$ as a function of the yield stress $\sigma_{y l d}$, and the effective porosity $f^{*}$.

$$
\Phi=\left(\frac{\sigma_{e q}}{\sigma_{y l d}}\right)^{2}+2 q_{1} f * \cosh \left(\frac{3}{2} q_{2} \frac{\sigma_{b y d d}}{\sigma_{y l d}}\right)-1-q_{1}^{2} f^{2}=0
$$

In which $q_{1}, q_{2}$ and $q_{3}$ are constitutive material parameters with $q_{3}=q_{1}^{2}, \sigma_{b y d}$ is the hydrostatic stress, and $\sigma_{e q}$ is the equivalent stress. The effective porosity $f^{*}$ in the equation above is defined as:

$$
f^{*}=\left\{\begin{array}{ccc}
f & \text { if } & f \leq f_{c} \\
f_{c}+\frac{\bar{f}_{F}-f_{c}}{\left(f_{F}-f_{c}\right)}\left(f-f_{c}\right) & \text { if } & f_{c}<f<f_{F} \\
\bar{f}_{F} & \text { if } & f \geq f_{F}
\end{array}\right.
$$

In this definition, the effective void volume fraction $f^{*}$, is a function of the void volume fraction $f$. The critical void volume fraction $f_{c}$, indicates the onset of void coalescence and parameter $f_{F}$ represents the void volume fraction at final failure. Constant $\bar{f}_{F}=1 / q_{1}$ is the maximum reachable value of the void volume fraction.

The evolution of void volume fraction, $\dot{f}$, can be considered as a combination of existing void growth, $\dot{f}_{\text {growth }}$, and nucleation of new voids, $\dot{f}_{\text {nucleation }}$.

$$
\dot{f}=\dot{f}_{\text {growth }}+\dot{f}_{\text {nucleation }}
$$


Void growth can be written as a function of the rate of plastic volume change, $\dot{\varepsilon}_{k k}^{p l}$.

$$
\dot{f}_{\text {growth }}=(1-f) \dot{\varepsilon}_{k k}^{p l}
$$

Void nucleation is defined in a strain-controlled nucleation function that considers a normal distribution for the nucleation strain. Consequently, the void nucleation rate can be written as:

$$
\dot{f}_{\text {nucleation }}=\frac{f_{N}}{s_{N \sqrt{2 \pi}}} \exp \left[-\frac{1}{2}\left(\frac{\bar{\varepsilon}^{p l}-\varepsilon_{N}}{s_{N}}\right)^{2}\right] \dot{\bar{\varepsilon}}^{p l}
$$

where $f_{N}$ defines the void volume fraction of nucleated voids, $\varepsilon_{N}$ and $s_{N}$ indicate the mean value and standard deviation of the nucleation strain respectively, $\bar{\varepsilon}^{p l}$ indicates the equivalent plastic strain, and $\dot{\bar{\varepsilon}}^{p l}$ defines the equivalent plastic strain rate. Finally, the initial void volume fraction $f_{0}$ indicates the presence of initial voids and is a measure for the relative density of the material.

Multiple studies have reported on the use of the GTN damage parameters for X70 and X100 grade pipeline steels [9, 10, 11, 12]. In this investigation, the GTN damage parameters, listed in Tab. 1, were considered as typical material constants which were obtained from literature.

\begin{tabular}{lcccccccc}
\hline Material & $q_{1}$ & $q_{2}$ & $f_{0}$ & $f_{C}$ & $f_{F}$ & $f_{N}$ & $e_{N}$ & $s_{N}$ \\
$\mathrm{X} 70$ & 1.5 & 1.0 & 0.000401 & 0.001517 & 0.5 & 0.067143 & 0.8 & 0.1 \\
$\mathrm{X} 100$ & 1.5 & 1.0 & 0.00015 & 0.02 & 0.18 & 0.005 & 0.3 & 0.1 \\
\hline
\end{tabular}

Table 1: GTN damage parameters for X70 and X100.

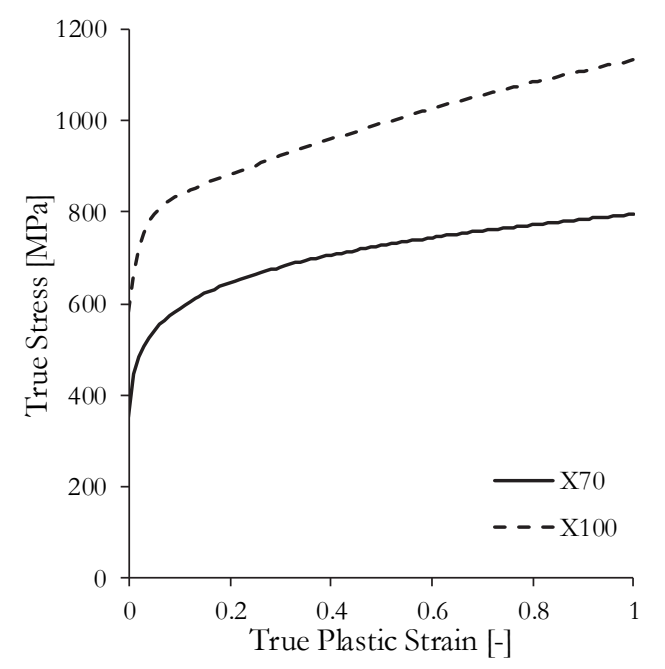

Figure 1: True stress-strain curve for X70 and X100 grade steels.

\section{Material Properties}

he mechanical properties of the investigated X70 and X100 grade steels were obtained from the literature [2,3]. The flow curves - as presented in Fig. 1 - describe the plasticity based on the true stress-strain relations. Two different isotropic hardening laws were used to define the material behaviour in the post-necking region. For X70 grade steel, 
a simple power law relation is defined. In case of X100 grade steel, a more complex isotropic relation was applied [13] as given in Tab. 2.

Strain rate dependency was taken into consideration using a Norton law, the plastic strain rate $\dot{\varepsilon}^{p l}$ is defined as:

$$
\dot{\varepsilon}^{p l}=\left(\frac{\sigma_{y l d}-R(\varepsilon)}{K}\right)^{n}
$$

Where $K$ and $n$ are referred to as the Norton parameters which were obtained from literature [3,10]. It should be noted that neither anisotropy nor adiabatic heating effects were taking into account throughout the numerical investigation. Tab. 2 gives an overview of the mechanical properties for X70 and X100 grade steel that were used for the numerical investigations.

\begin{tabular}{|c|c|c|c|}
\hline & & $\mathrm{X70}$ & X100 \\
\hline Young's modulus & $E$ & $210 \mathrm{GPa}$ & $210 \mathrm{GPa}$ \\
\hline Poisson's ratio & $v$ & 0.3 & 0.3 \\
\hline $\begin{array}{l}\text { Isotropic } \\
\text { hardening }\end{array}$ & $\begin{array}{c}R(\varepsilon)=R_{0}\left(\varepsilon^{p l}+\varepsilon_{0}\right)^{n} \\
R(\varepsilon)=R_{0}\left(1+Q_{1}\left(1-e^{-k_{1} \varepsilon^{p^{l}}}\right)+Q_{2}\left(1-e^{-k_{2} \varepsilon^{p l}}\right)\right)\end{array}$ & $\begin{array}{c}\mathrm{R}_{0}=795 \\
\quad M P a \\
n=0.13 \\
\varepsilon_{0}=0.002\end{array}$ & $\begin{array}{c}R_{0}=580 \\
M P a \\
Q_{1}=0.367 \\
Q_{2}=1.119 \\
k_{1}=46.48 \\
k_{2}=0.7411\end{array}$ \\
\hline Strain rate effect & $\begin{array}{l}K \\
n\end{array}$ & $\begin{array}{c}55 \mathrm{MPa} s^{1 / n} \\
5\end{array}$ & $\begin{array}{c}55 \mathrm{MPa} s^{1 / n} \\
5\end{array}$ \\
\hline
\end{tabular}

Table 2: Mechanical material properties for X70 and X100 grade steels.

\section{NUMERICAL MODELS}

$\mathrm{F}$ or each lab-scale fracture toughness experiment (CVN, DWT'T and DT3), a 3D solid finite element model was constructed using the ABAQUS software package. The ABAQUS/Explicit solver which includes the standard GTN model referred to as "porous metal plasticity" was used in each model to simulate the dynamic fracture propagation. The geometry was created based on the standard specimen dimensions as previously discussed. In each model, the mesh was created using linear 8-node brick elements with reduced integration. The mesh size has a significant importance when the GTN damage model is implemented. A structured partitioning technique was applied using Python scripting methodology as to keep the computational effort to an acceptable level. This meshing strategy was applied for each model and allowed for a uniform and fine mesh size in the region of crack propagation whilst larger elements could be used for the remainder. As reported in literature, the element size perpendicular to the crack propagation direction is dependent on the microstructure of the material and should range between $0.05 \mathrm{~mm}$ and $0.30 \mathrm{~mm}$ for ferritic steels $[14,15,16]$. In this study an element size of $0.2 \mathrm{~mm}$ was used. Due to the large-scale geometry of the DT3, the mesh size in the crack region was increased to $0.7 \mathrm{~mm}$ to keep computational efforts acceptable. For both the CVN test and DWTT, the anvil and striker were modelled using rigid elements. An overview of the geometry of the constructed numerical models and their respective meshing strategy is presented in Fig. 2. In the case of the CVN and DWT'T models, a "hard" contact definition using a zero-penetration condition between hammer and specimen was implemented. Also, friction was taken into consideration using a penalty function with friction coefficient 0.1 . Due to the impact loading in the CVN and DWTT, acceleration and consequently force measurements can show pronounced oscillations. Therefore, velocity data was extracted and derived to obtain acceleration and force data. This method reduced the presence of oscillations and allowed to construct the forcedisplacement curves for each respective simulation. 


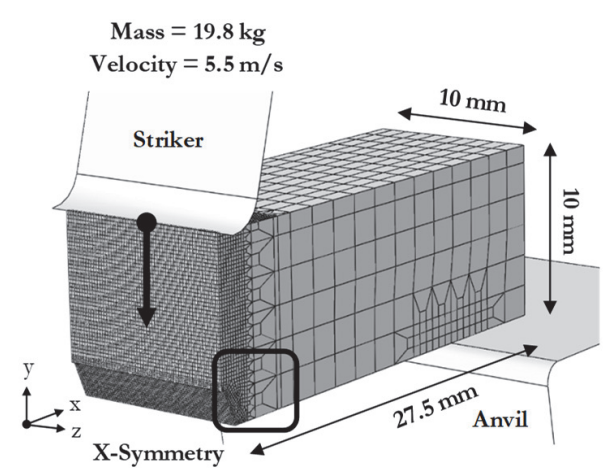

(a)
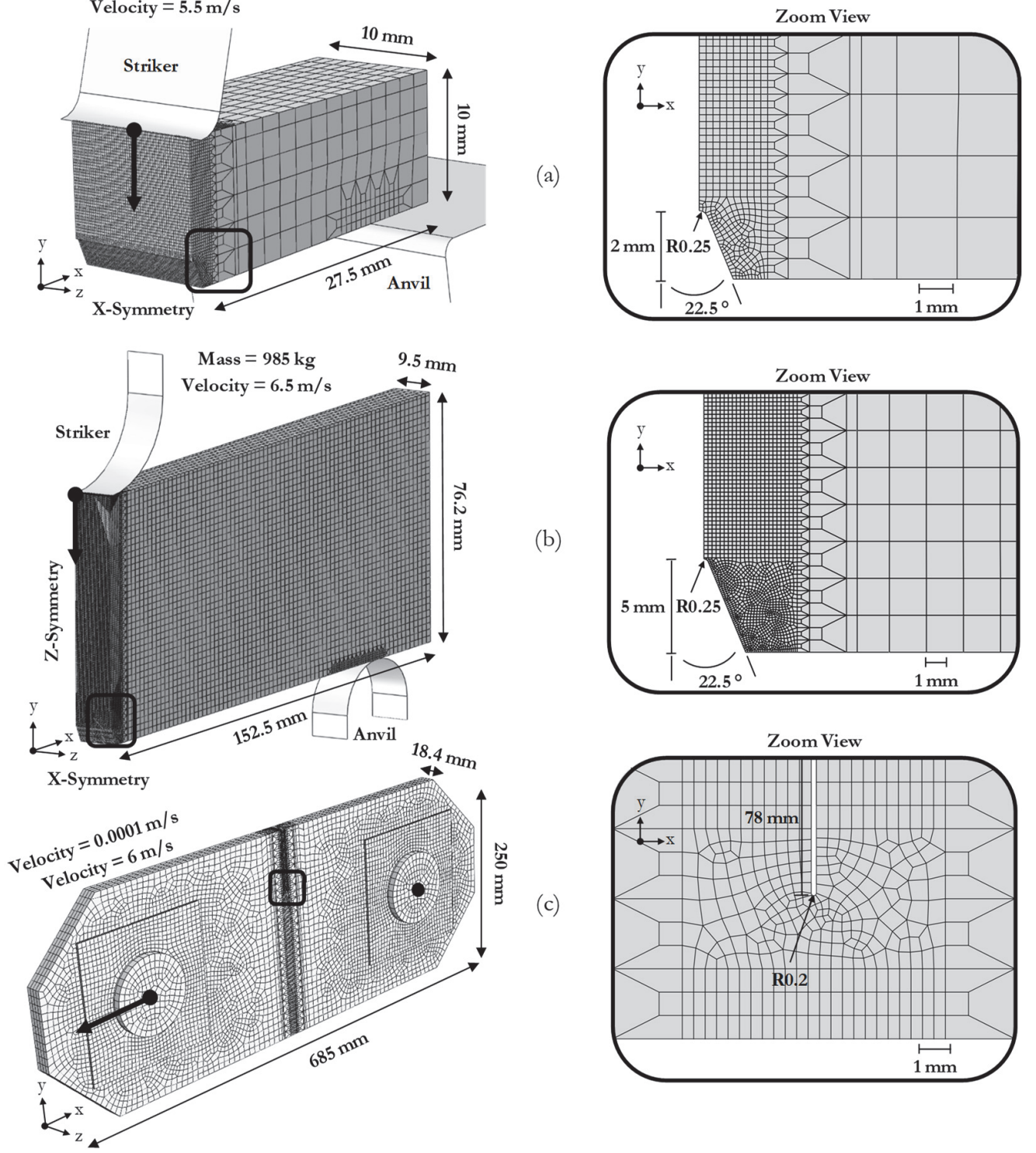

(b)
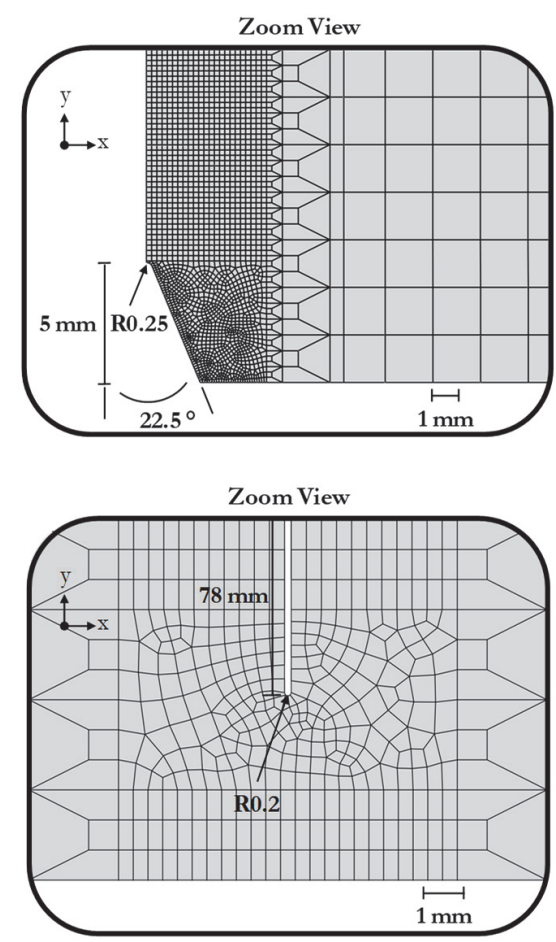

Figure 2: Specimen geometry, notch geometry, boundary conditions, and meshing strategy for CVN specimen (a), DWTT specimen (b), and DT3 specimen (c).

\section{CVNMODEL}

One half of a standard CVN specimen with a cross section of $10 \mathrm{~mm} \times 10 \mathrm{~mm}$ and length of $27.5 \mathrm{~mm}$ was constructed. As to replicate the hammer impact on the specimen, an initial velocity of $5.5 \mathrm{~m} / \mathrm{s}$ and a mass of $19.8 \mathrm{~kg}$ were assigned to the reference point of the hammer [17]. Due to the geometry, symmetry conditions were applied on the Y-Z plane. For this model, X70 grade material properties were assigned to the specimen.

\section{DWTT MODEL}

The geometry of the modelled specimen measures a cross section of $9.5 \mathrm{~mm} \times 76.2 \mathrm{~mm}$ and a length of $152.5 \mathrm{~mm}$. The larger dimensions resulted in a larger number of elements, thus an extra symmetry condition was applied. Consequently, a total of two symmetry planes were defined on the X-Y plane and on the Y-Z plane of the specimen, and only one quarter of a standard DW'T specimen was constructed. According to the performed DWTT experiments, an initial velocity of 6.5 $\mathrm{m} / \mathrm{s}$ and a mass of $985 \mathrm{~kg}$ were assigned to the reference point of the striker. For the DWTT model, X70 material properties were assigned to the specimen. 


\section{DT3 MODEL}

The DT3 specimen has a significantly larger size than the previously discussed models with a cross section of $18.4 \mathrm{~mm} \times$ $250 \mathrm{~mm}$ and length of $685 \mathrm{~mm}$. Furthermore, no symmetry conditions could be applied since the boundary conditions and loading conditions cannot be considered symmetrical. Each side of the specimen is connected to a rail driven frame using a solid steel pin. One pin is assumed to be constrained in every translation direction whilst the other pin is able to move in the loading direction. Each pin was modelled using rigid elements. The DT3 test procedure consists out of a quasi-static phase in which hydraulic cylinders apply a tensile load onto the specimen. During this loading phase, springs are compressed, and the potential energy is stored. Once a crack initiates, the system becomes unstable and the springs release their potential energy. To simulate the quasi-static and dynamic phases, a velocity of $0.0001 \mathrm{~m} / \mathrm{s}$ and $6 \mathrm{~m} / \mathrm{s}$ was assigned to one of the pins, respectively. These pin velocities where calculated based on the experimental displacement-time measurements described by Luu [3]. The instant transition from a quasi-static to dynamic velocity is physically not feasible. However, based on the obtained numerical results, the instant transition approximation can be considered satisfactory. Due to the large time differences (180 s for the quasi-static phase and $10 \mathrm{~ms}$ for the dynamic phase), a different modelling approach was used. The simulation was split into two models: an implicit model simulating the quasi-static displacement of the hydraulic cylinders, and an explicit model simulating the crack propagation initiated by the springs. Subsequently, the implicit model state was used to define the initial state for the following explicit simulation. In this case, X100 material properties were assigned to the specimen as well as the reinforcements.

\section{RESUlTS AND DisCUSSION}

he experimental load-displacement curve and the corresponding numerical prediction for each of the 3 tests are shown in Fig. 3. For the CVN simulation shown in Fig. 3 (a), a good correlation with the experimental data [17] was obtained. In the case of the DWTT, experimental data was obtained through tests on an instrumented DWTT experiment at room temperature. As can be seen in Fig. 3 (b), the DWTT simulation also shows a good agreement with these experiments. However, a force peak is observed in the simulation at the moment of crack initiation. Fig. 3 (c) shows the correlation of the DT3 simulation with experimental data. Even though the mesh size was increased for the DT3 simulation, a very good correlation with experimental data was obtained, especially in the dynamic crack propagation phase. Also, acceleration and force measurements showed considerably less oscillation than in the CVN and DWTT models.

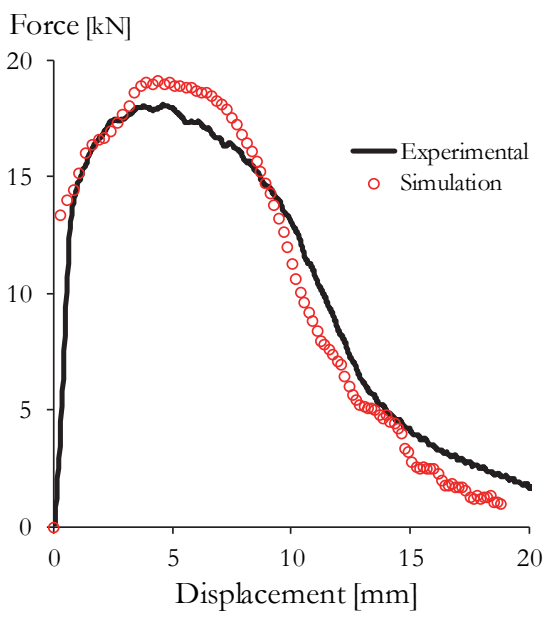

(a)

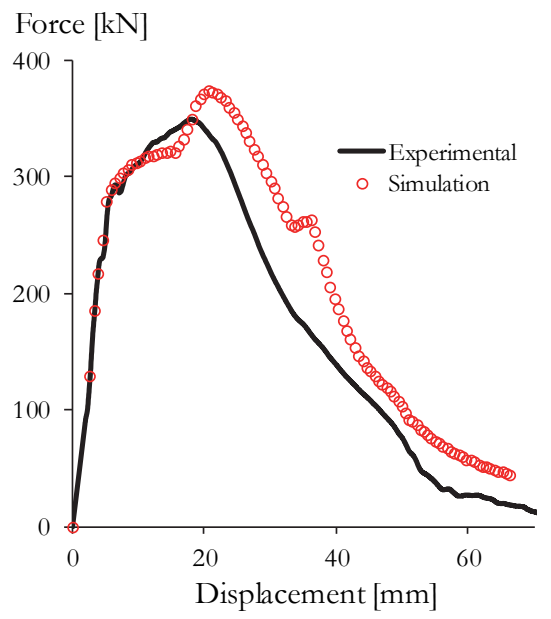

(b)

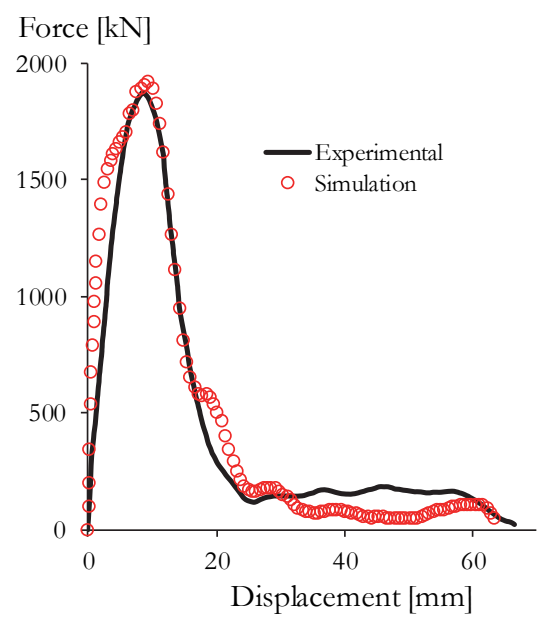

(c)

Figure 3: Experimental and numerical force-displacement curves for the CVN experiment (a), the DWTT experiment (b) and the DT3 experiment (c).

Fig. 4 gives an overview of the resulting fracture surfaces, $\mathrm{MPa}$ is used as a unit for the displayed stress distributions. Comparing the CVN and DWTT fracture surface presented in Fig. 4 (a) and Fig. 4 (b) respectively, the same characteristic features can be observed. In both cases, thickness reduction appears at the initial notch and a thickness increase at the end of the fracture. Besides these two similarities, the remainder of the fracture surface actually differs from each other. The central part of the observed DWT'T fracture surface is more related to the DT3 fracture surface represented in Fig. 4 (c). 
Again, a thickness reduction at the initial notch and a thickness increase at the end of the fracture is observed in the DT3 specimen. In this case, the stress concentrations in the centre of the fracture are similar to the stress concentrations observed in the DWTT specimen.

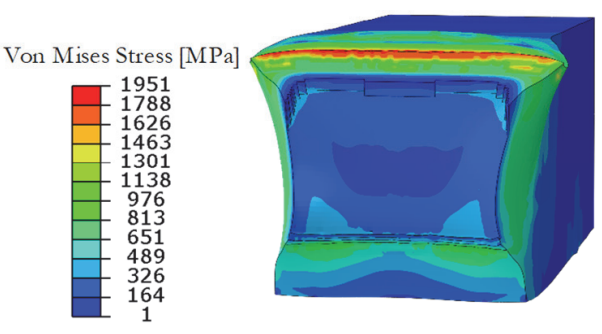

(a)

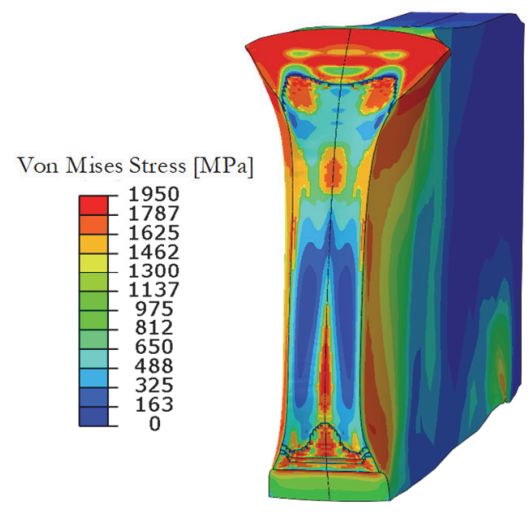

(b)

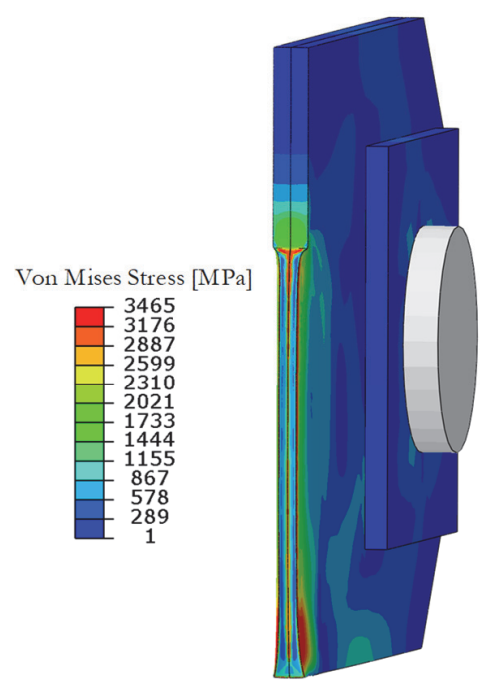

(c)

Figure 4: Fracture surfaces and corresponding stress distributions obtained through numerical simulations for the CVN specimen (a), the DWTT specimen (b), and the DT3 specimen (c).

As can be observed in Fig. 3, the peak force prediction of the CVN simulation and DWTT simulation is overestimated in comparison with the experimental data. In contrast, the DT3 peak force estimation does approximate the experimental data rather accurate. This observation suggests that the presence of impact loading in the numerical model increasingly complicates the acceleration and force calculations. As the impact conditions change during the simulation, the strain rate will also be adjusted continuously. Consequently, the explicit integration and the constitutive material model will be affected. The DT3 test on the other hand, only uses a tensile force to introduce the crack propagation leading to an overall more stable simulation. Furthermore, as to fulfil the GTN mesh size requirement, the element dimensions for the differently scaled CVN model and DW'T'T model were kept constant. This mesh size with respect to the total scale of the model will also influence the explicit solution scheme. Moreover, relatively small elements are required for the GTN model, thus the computational effort significantly increases due to the fine meshing. Therefore, the relatively larger geometry of the specimen does augment the issue regarding the contradictory role of the mesh size as material parameter as well as FE parameter. It is noteworthy that, even though the element size of the DT3 specimen was increased, the ductile fracture propagation does still present a good correlation with the experimental data.

\section{CONCLUSIONS}

7 he main objective of this paper was to compare the numerical modelling of dynamic ductile fracture propagation on three different testing scales using the GTN damage model. Based on the obtained data, following conclusions can be drawn:

- In this study, the implemented GTN ductile damage model is able to describe ductile fracture propagation on a satisfactory level for the considered different lab-scale fracture tests using high-toughness grade pipeline steels with different mechanical properties.

- The presence of impact loading does complicate the force prediction especially when the size of the considered specimen increases. Even though, the test mechanism is similar and stress-strain conditions are the same, it is clear that the simulation of the DWTT is less stable than the smaller CVN test.

- Due to the large scale of the DT3 specimen, it currently is challenging to respect the mesh size requirements for the GTN damage model. The mesh size plays a contradictory role as a material property as well as a fundamental 
finite element property. However, even without fulfilling the mesh size requirement, the DT3 simulation did approximate experimental data accurately.

In future investigations, more advanced damage materials models will be implemented to improve the predictive performance of the numerical simulations. Furthermore, the GTN relations only considers isotropic ductile damage conditions as well as the physical mechanisms of ductile damage evolution. In order to include brittle damage, multiple extensions of the GTN model have been proposed. Some suggestions include a non-local probability approach; others are based on a clear brittle fracture criterion. However, these proposed models increase the calibration efforts considerably. Therefore, in future research more advanced numerical models are required as to implement anisotropic behaviour as well as temperature dependency without overcomplicating the calibration procedures.

\section{ACKNOWLEDGEMENTS}

he author gratefully acknowledges the support of the Research Foundation Flanders (FWO) via PhD fellowship grant $1 \mathrm{SB} 6420 \mathrm{~N} .$.

\section{REFERENCES}

[1] Zhu, X. K. and Leis, B. N. (2013). Ductile fracture arrest methods for gas transmission pipelines using Charpy impact energy or DW'T'T energy, Journal of Pipeline Engineering, 3(12), pp. 259-272.

[2] Rivalin, F., Pineau, A., Di Fant, M. and Besson, J. (2001). Ductile tearing of pipeline-steel wide plates I. Dynamic and quasi-static experiments, Engineering Fracture Mechanics, 68, pp. 329-345.

[3] Luu, T. T. (2006). Déchirure ductile des aciers à haute résistance pour gazoducs (X100), École Nationale Supérieure des Mines de Paris, Paris.

[4] ASTM International, (2019). ASTM A370 -19e1, Standard Test Methods and Definitions for Mechanical Testing of Steel Products, West Conshohocken, PA.

[5] Mannucci, G., Demofonti, G. and Di Biagio, M. (2005). X100 - Fracture initiation and propagation.

[6] Zhu, X. K. (2015). State-of-the-art review of fracture control technology for modern and vintage gas transmission pipelines, Engineering Fracture Mechanics, 148, pp. 260-280.

[7] Chu, C. C. and Needleman, A. (1980). Void Nucleation Effects in Biaxially Streched Sheets, Journal of Engineering Materials and Technology, 3(102), pp. 249-256.

[8] Tvergaard, V. and Needleman, A. (1984). Analysis of the cup-cone fractured in a round tensile bar, Acta Metallurgica, 1(32), pp. 157-169.

[9] Maxey, W. A., Kiefner, J. F., Eiber, R. J. and Duffy, A. R. (1971). Ductile fracture initiation, propagation, and arrest in cylindrical vessels, in Proceedings of the National Symposium on Fracture Mechanics Part II. ASTM STP 514, Illinois.

[10] Rivalin, F., Besson, J., Pineau, A. and Di Fant, M. (2001), Ductile tearing of pipeline-steel wide plates II. Modeling of in-plane crack propagation, Engineering Fracture Mechanics, no. 68, pp. 347-364.

[11] Nonn, A. and Kalwa, C. (2012). Simulation of ductile crack propagation in high-strength pipeline steel using damage models, in Proceedings of the 9th International Pipeline Conference, Calgary.

[12] Thibaux, P. and Van den Abeele, F. (2009). Determination of crack initiation and propagation energy in instrumented Charpy V-notch impact tests by finite element simulations, in Pipeline Technology Conference, Ostend.

[13] Besson, J.,Cailletaud, G., Chaboche, J.-L. and Forest, S. (2001). Mécanique non linéaire des matériaux, Paris: Hermes.

[14] Ruggieri, C., Dodds, R. H. and Panontin, T. L. (1996). Numerical Modeling of Ductile Crack Growth in 3-D Using Computational Cell Elements, Department of Civil Engineering, University of Illinois, Illinois.

[15] Chen, Y. and Lambert, S. (2003). Analysis of ductile tearing of pipeline-steel in single edge notch tension specimen, International Journal of Fracture, 124, pp. 179-199.

[16] Scheider, I., Nonn, A., Völling, A., Mondry, A.and Kalwa, C. (2014). A damage mechanics based evaluation of dynamic fracture resistance in gas pipelines, Procedia Materials Science, 3, pp. 1956-1964.

[17] Talemi, R., Cooreman, S., Mahgerefteh, H., Martynov, S. and Brown, S. (2019). A fully coupled fluid-structure interaction simulation of three-dimensional dynamic ductile fracture in a steel pipeline, Theoretical and Applied Fracture Mechanics, 101, pp. 224-235. 